\title{
Lysophosphatidic acid activates $\beta$-catenin/T cell factor signaling, which contributes to the suppression of apoptosis in H19-7 cells
}

\author{
YUANJIE SUN ${ }^{1}$, NAM-HO KIM ${ }^{2}$, LITING JI ${ }^{3}$, SEUNG-HYUK KIM ${ }^{2}$, JONGHO LEE $^{2}$ and HAE JIN RHEE ${ }^{1,3}$ \\ ${ }^{1}$ Institute of Natural Medicine, and Departments of ${ }^{2}$ Pharmacology and ${ }^{3}$ Natural Medicine, College of Medicine, \\ Hallym University, Chuncheon, Gangwon-do 200-702, Republic of Korea
}

Received March 26, 2013; Accepted October 3, 2013

DOI: $10.3892 / \mathrm{mmr} .2013 .1743$

\begin{abstract}
Lysophosphatidic acid (LPA) is a lipid growth factor that regulates diverse cell functions, including cell proliferation, survival and apoptosis. LPA has been demonstrated to be involved in the regulation of cortical neurogenesis by increasing the survival of neural precursors. Previously, we reported that LPA stimulated the inactivation of glycogen synthase kinase 3 (GSK3) via the $\mathrm{G}$ protein-coupled $\mathrm{LPA}_{1}$ and $\mathrm{LPA}_{2}$ receptors, by which apoptosis is suppressed in H19-7 cells [an embryonic hippocampal progenitor cell (HPC) line]. Increasing numbers of studies have demonstrated that certain $\mathrm{G}$ protein-coupled receptors activate $\beta$-catenin/T cell factor (TCF) signaling independently of Wnt, which is involved in cell fate determination, cell proliferation and cell survival. To determine whether LPA activates $\beta$-catenin-mediated transcriptional activation pathways and whether $\beta$-catenin/TCF signaling is involved in neurogenesis by controlling the survival of neural precursors, $\beta$-catenin/TCF signaling cascades induced by LPA were investigated in the HPCs. Activation of $\beta$-catenin/TCF signaling was determined by the nuclear translocation of $\beta$-catenin and the transcriptional activation of a TCF reporter gene. The activation of $\beta$-catenin/TCF signaling was blocked by pertussis toxin (PTX) and a protein kinase $\mathrm{C}$ (PKC) inhibitor. The expression of a constitutively active mutated form of GSK $3 \beta$ activated $\beta$-catenin/TCF signaling to comparable levels to those induced by LPA, and protected against apoptosis in differentiating H19-7 cells. These results showed that LPA activates $\beta$-catenin/TCF signaling in a PTX- and
\end{abstract}

Correspondence to: Professor Hae Jin Rhee, Institute of Natural Medicine, College of Medicine, Hallym University, Hallymdaehak-gil 1, Chuncheon, Gangwon-do, 200-702, Republic of Korea

E-mail: hjrhee@hallym.ac.kr

Professor Jongho Lee, Department of Pharmacology, College of Medicine, Hallym University, Hallymdaehak-gil 1, Chunchon, Kangwon-do 200-702, Republic of Korea

E-mail: jongholee@hallym.ac.kr

Key words: lysophosphatidic acid, $\beta$-catenin, $\mathrm{T}$ cell factor, H19-7 cells, glycogen synthase kinase 3, survival
PKC-dependent manner, which contributes to LPA-induced cell survival in the HPCs. Activation of $\beta$-catenin/TCF signaling by LPA may be involved in neurogenesis by controlling the survival of neural precursors.

\section{Introduction}

Lysophosphatidic acid (LPA) affects various cell functions, including cell migration, proliferation, differentiation and survival, through a family of cognate G-protein coupled receptors (GPCRs). The binding of LPA to its cognate receptors elicits a range of LPA downstream signaling through at least three different types of $G$ proteins, including Gi/o, Gq/11 and G12/13 (1). LPA is also involved in diverse neurodevelopmental processes, such as neurogenesis, neuritogenesis and neuronal migration. We have previously demonstrated that LPA protected differentiating hippocampal progenitor cells (HPCs) from apoptosis by inhibiting the phosphorylation of glycogen synthase kinase 3 (GSK3) (2), suggesting that LPA may be involved in hippocampal neurogenesis. GSK3 plays a central role in the canonical Wnt/ $\beta$-catenin signaling pathway, which is shown to be important in neurogenesis by supporting the proliferation of neural stem and precursor cells (3). Previous studies have demonstrated novel aspects of Wnt $/ \beta$-catenin signaling in neurogenesis. Wnt $/ \beta$-catenin signaling is involved in neurogenesis by regulating the differentiation of neural precursors (4-7). Moreover, studies have revealed that $\beta$-catenin signaling promotes the survival of neuronal cells, including precursor cells (8-12). This has led to the hypothesis that $\beta$-catenin signaling may regulate neurogenesis by modulating the survival of neuronal progenitor cells (NPCs). LPA is also involved in the regulation of cortical neurogenesis by controlling the survival of NPCs in ex vivo cortical culture systems, although the molecular mechanism for this is not fully understood (13). Increasing data demonstrate that the activation of $\mathrm{G}$ proteins alone, or the activation of GPCRs by agonists such as lipid metabolites and growth factors, may induce the stabilization of $\beta$-catenin and the subsequent $\beta$-catenin/T cell factor (TCF) transcriptional activation that is independent of Wnt $(14,15)$.

In the present study, it was investigated whether LPA induced $\beta$-catenin/TCF transcriptional activation and whether 
$\beta$-catenin/TCF signaling by LPA was involved in the control of NPC survival. It was demonstrated that LPA activated the $\beta$-catenin/TCF signaling predominantly in Gi/o- and protein kinase $\mathrm{C}$ (PKC)-dependent pathways, and that the $\beta$-catenin/TCF signaling induced by LPA was involved in the suppression of apoptosis in differentiating H19-7 cells. Therefore, the activation of the $\beta$-catenin/TCF signaling pathway by LPA may function in neurogenesis by controlling the survival of neural precursors.

\section{Materials and methods}

Materials. H19-7 cells were generously provided by Dr. Kwang C. Chung (Yonsei University, Seoul, Republic of Korea). LPA (1-oleoyl-2-hydroxy-sn-glycero-3-phosphate), 5-bromodeoxyuridine (BrdU), fatty acid-free bovine serum albumin and pertussis toxin (PTX) were purchased from Sigma (St. Louis, MO, USA). Wortmannin and GF109203 were purchased from Tocris Bioscience (Bristol, UK). TOP Flash and FOP Flash luciferase reporters were purchased from Upstate Biotechnology (Lake Placid, NY, USA). Alexa Fluor 568-conjugated Annexin $\mathrm{V}$ was purchased from Invitrogen Life Technologies (Carlsbad, CA, USA). Dulbecco's modified Eagle's medium (DMEM), fetal bovine serum (FBS) and G418 were obtained from Invitrogen Life Technologies. All other reagents were of analytical grade or the highest purity available.

Cell culture. H19-7 cells, conditionally immortalized, embryonic (day 17) rat hippocampal cells with a temperature-sensitive SV40 large T antigen (16), were maintained at $33^{\circ} \mathrm{C}$ (the permissive temperature at which SV40 large $\mathrm{T}$ is functional) in DMEM supplemented with $10 \% \mathrm{FBS}$, $0.2 \mathrm{mg} / \mathrm{ml} \mathrm{G} 418,50 \mathrm{U} / \mathrm{ml}$ penicillin and $50 \mathrm{mg} / \mathrm{ml}$ streptomycin. For differentiation, H19-7 cells were shifted to $39^{\circ} \mathrm{C}$ to eliminate the expression of the functional large $\mathrm{T}$ antigen, then incubated for $24 \mathrm{~h}$ in DMEM supplemented with N2 (N2 medium), and treated with LPA. The differentiation of H19-7 cells into neuronal cells was confirmed by the expression of neuronal markers, such as neurofilament-M and neuron specific enolase, and cell morphology as previously described (17). To determine effect of pharmacological inhibitors on the TCF transcriptional activation and inhibitory phosphorylation of GSK3 $\beta$ induced by LPA, the H19-7 cells were pretreated with the inhibitor for $1 \mathrm{~h}$ before LPA treatment.

Measurement of apoptosis by Annexin V staining. Apoptosis was measured by staining with Annexin V conjugated to Alexa Fluor 568 (Life Technologies, Grand Island, NY, USA) as previously described (2). H19-7 cells were collected, washed with phosphate-buffered saline (PBS) and resuspended in $100 \mu \mathrm{l}$ binding buffer [10 mM HEPES (pH 7.4), $140 \mathrm{mM} \mathrm{NaCl}$ and $2.5 \mathrm{mM} \mathrm{CaCl}_{2}$ ]. The cells were then incubated with $5 \mu \mathrm{l}$ Annexin V-Alexa Fluor 568 for $15 \mathrm{~min}$ at room temperature in the dark, followed by the addition of $400 \mu \mathrm{l}$ binding buffer. The cells were analyzed by flow cytometry using a Guava EasyCyte (GE Healthcare, Piscataway, NJ, USA).

Western blot analysis. H19-7 cells were collected by brief centrifugation at $500 \mathrm{x} \mathrm{g}$ and resuspended in lysis buffer
[10 mM HEPES (pH 7.5), 10 mM KCl, 1 mM EGTA, $1 \mathrm{mM}$ EDTA, $1 \%$ Nonidet P-40, $1 \mathrm{mM} \mathrm{Na}_{3} \mathrm{VO}_{4}, 5 \mathrm{mM} \mathrm{NaF}$ and protease inhibitor cocktail]. Following incubation on ice for $10 \mathrm{~min}$, cell lysates were centrifuged at 4,000 x $\mathrm{g}$ for $1 \mathrm{~min}$. Supernatants containing cytoplasm were collected and the remaining nuclear pellet was resuspended in lysis buffer containing $0.1 \%$ sodium dodecyl sulphate (SDS). The protein concentrations within each lysate were determined by the Bradford assay (Bio-Rad, Richmond, CA, USA). The fractionated extracts were mixed with SDS sample buffer and equal quantities of the samples were loaded and separated by SDS-polyacrylamide gel electrophoresis (8 or 10\% reducing gels), transferred to polyvinylidene difluoride membranes (Millipore, Bedford, MA, USA) and blocked with 5\% non-fat milk. The membranes were incubated in primary antibody overnight at $4^{\circ} \mathrm{C}$. Subsequent to this, the membranes were washed in TBST [10 $\mathrm{mM}$ Tris, $140 \mathrm{mM} \mathrm{NaCl}$ and $0.1 \%$ Tween-20 ( $\mathrm{pH} 7.6$ )], incubated with the appropriate secondary antibody and washed again in TBST. Bands were visualized by enhanced chemiluminescence and exposed to X-ray film.

Immunocytochemistry. Differentiating H19-7 cells cultivated on chamber slides (Nalgene, Rochester, NY, USA) were treated with LPA. The cells were fixed and permeabilized as described in a previous study (2), processed for immunocytochemistry with mouse anti- $\beta$-tublin III (Sigma) or rabbit anti- $\beta$-catenin (Abcam, Cambridge, MA, USA), and subsequently processed with Alexa Fluor 568- or Alexa Fluor 488-conjugated secondary antibodies (Jackson ImmunoResearch, West Grove, PA, USA). The stained cells were observed using a fluorescence microscope (Axio Scope A1; Zeiss, Oberkochen, Germany) with a digital camera attachment.

Luciferase reporter gene assay. H19-7 cells were transiently transfected with a luciferase reporter and Renilla luciferase (pRL-TK; Promega Corporation, Madisson, WI, USA) with the indicated plasmids, using FuGene HD (Roche Molecular Biochemicals, Mannheim Germany). The luciferase reporter constructs TOP Flash and FOP Flash (Upstate Biotechnology) were used to determine the $\mathrm{TCF} / \mathrm{lym}$ hoid enhancing factor (LEF) transcription activity. Following transfection for $6 \mathrm{~h}$, the cells were cultivated in N2 medium for $18 \mathrm{~h}$ and to induce differentiation as described previously. The cells were treated with LPA or vehicle for $4 \mathrm{~h}$ and collected. The cell extracts were prepared by rinsing each plate twice with PBS and lysing the cells in $150 \mu$ l Reporter Assay Lysis buffer (Promega Corporation). The cell lysates were collected, normalized for protein content and assayed using a dual luciferase assay system (Promega Corporation). They were analyzed for firefly luciferase (TOP Flash or FOP Flash) activity, and the reactions were quenched and immediately reanalyzed for Renilla luciferase activity using an Autolumat Luminometer (Berthhold Technologies, Oak Ridge, TN, USA).

Statistical analysis. Results are presented as the mean \pm SEM. Statistical significance was analyzed by one-way analysis of variance followed by a Dunnett's multiple test or a paired t-test using Prism (GradPad Software, La Jolla, CA, USA). P<0.05 was considered to indicate a statistically significant difference. 


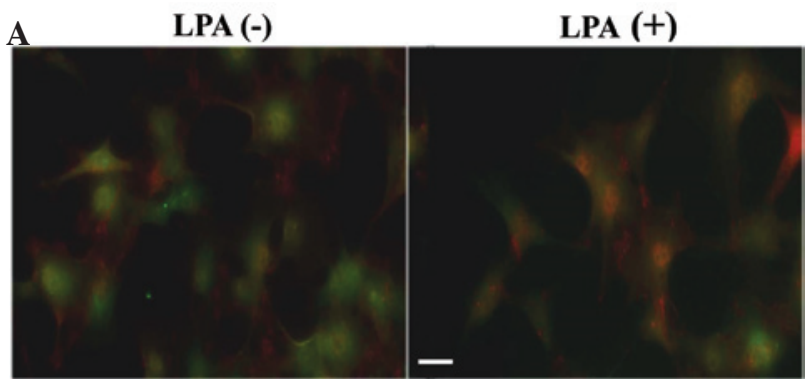

B
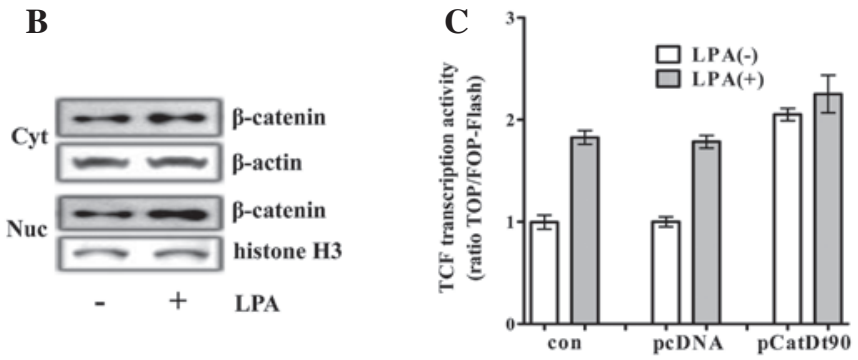

Figure 1.Lysophosphatidic acid (LPA) activated $\beta$-catenin/T cell factor (TCF) signaling in differentiating H19-7 cells. H19-7 cells were induced to differentiate and were treated with $5 \mu \mathrm{M}$ LPA or vehicle. (A) The cells were fixed, immunostained with anti- $\beta$-catenin or anti-tublin III and detected with secondary antibody conjugated with Alexa Fluor 568 and Alexa Fluor 488, respectively. Scale bar, $10 \mu \mathrm{m}$. (B) The cells were lysed and fractionated and the levels of $\beta$-catenin in the cytoplasm and nucleus were determined by western blot analysis. Representatives of two independent experiments are shown. (C) H19-7 cells were transfected with TOP Flash or POP Flash and pRL-TK, along with additional plasmid encoding CA- $\beta$-catenin or empty vector before inducing differentiation. The cells were treated with LPA or vehicle, and TCF reporter transcriptional activity was demonstrated by determining the ratio of TOP-Flash to POP-Flash, as described in the Materials and methods. Each point represents the mean \pm SEM of triplicate samples. Data are representative of three independent experiments.

\section{Results}

LPA induces activation of $\beta$-catenin/TCF signaling in H19-7 cells. In a previous study, it was demonstrated that LPA stimulated the inactivation of GSK-3 in H19-7 cells through $\mathrm{LPA}_{1}$ and $\mathrm{LPA}_{2}$ (2). As $\beta$-catenin is a substrate for GSK3 and is degraded following phosphorylation by GSK3, and as LPA activates $\mathrm{G}$ proteins, such as Gq, Gi/o and G12/13 (via coupling to $\mathrm{LPA}_{1}$ and $\mathrm{LPA}_{2}$ ), LPA may activate $\beta$-catenin/TCF signaling in H19-7 cells. To determine whether LPA activated $\beta$-catenin/TCF signaling, cytoplasmic and nuclear levels of $\beta$-catenin in the H19-7 cells treated with LPA were investigated by immunocytochemistry and western blot analysis. An increase in the level and the nuclear translocation of $\beta$-catenin was detected by immunocytochemistry in the H19-7 cells (Fig. 1A). In addition, an increase in the cytoplasmic levels of $\beta$-catenin and the subsequent increase in the levels of nuclear $\beta$-catenin was demonstrated by western blot analysis (Fig. 1B). An increase in the level of nuclear $\beta$-catenin was also detected within $30 \mathrm{~min}$ (data not shown). To determine whether LPA activated $\beta$-catenin-mediated transcriptional activation, the TCF reporter activity was analyzed in differentiating H19-7 cells transfected with TCF luciferase reporter. TCF with LPA reporter activity was $\sim 2$-fold that without LPA, and was similar to that induced by transfection with a plasmid encoding $\beta$-catenin truncated $\mathrm{N}$-terminal 90 amino acids

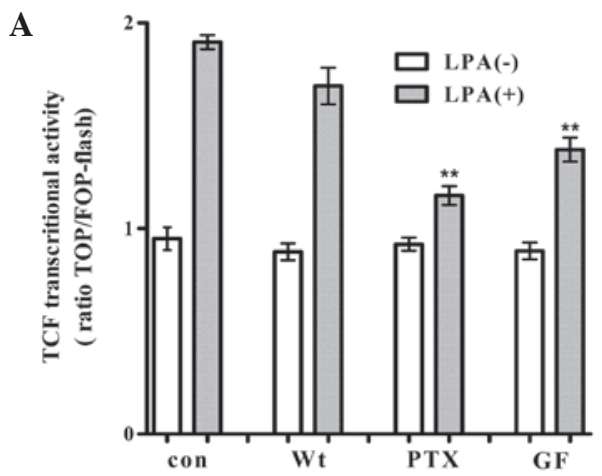

B

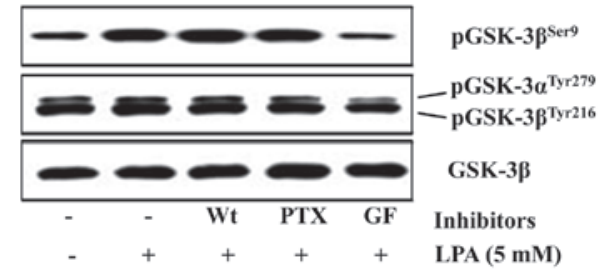

Figure 2. Effect of pharmacological inhibitors on the T cell factor (TCF) transcriptional activity and inhibitory phosphorylation of glycogen synthase kinase (GSK)3 $\beta$. (A) H19-7 cells were transiently transfected with TOP Flash or FOP Flash, and differentiation was induced. The differentiating cells were pretreated with $10 \mu \mathrm{M}$ GF109203,2 $\mu \mathrm{M}$ wortmannin and $100 \mathrm{ng} / \mathrm{ml}$ pertussis toxin (PTX) for $1 \mathrm{~h}$, and treated with LPA $(5 \mu \mathrm{M})$ or vehicle for $4 \mathrm{~h}$. The cells were collected and TCF reporter activity was determined. Results are presented as the mean of triplicate samples from three independent experiments. The value in the absence of inhibitor (control) versus value in the presence of inhibitor are significantly different $\left(\mathrm{n}=9,{ }^{* * *} \mathrm{P}<0.001\right)$. (B) Differentiating H19-7 cells were treated with $10 \mu \mathrm{M}$ GF109203, $2 \mu \mathrm{M}$ wortmannin and $100 \mathrm{ng} / \mathrm{ml}$ PTX for $1 \mathrm{~h}$, and treated with LPA $(5 \mu \mathrm{M})$ or vehicle for $4 \mathrm{~h}$. Inhibitory phosphorylation of GSK $3 \beta$ was detected using anti-GSK-3 $\beta$ Ser9 (Cell Signaling Technology Inc., Berverly, MA, USA) by western blot analysis. Data are representative of three independent experiments.

(b-Cat $\Delta 90$ ) acting as a constitutively active mutant of $\beta$-catenin (CA- $\beta$-catenin) (Fig. 1C).

LPA activates $\beta$-catenin/TCF signaling in a PTX-and $P K C$-dependent manner. Our previous study demonstrated that LPA induced cell survival in a PTX-dependent manner by the post-translational upregulation of Mcl-1 through the inhibitory phosphorylation of GSK3 in differentiating H19-7 cells. The inhibitory phosphorylation of GSK3 is mediated predominantly by $\mathrm{PKC}$ and partly by mitogen activated protein kinases, PKA and phosphatidylinositol 3-kinase (PI3K) in the H19-7 cells (2). To determine the mechanism by which LPA stimulates $\beta$-catenin/TCF signaling, the effect of pharmacological inhibitors of PKC, PI3K and Gi/o on the LPA-induced activation of the TCF reporter activity and on the inhibitory phosphorylation of GSK3 $\beta$ at Ser9 by LPA was analyzed. GF109203 (a PKC inhibitor) and PTX (a Gi/o specific inhibitor) significantly decreased the LPA-induced activation of TCF reporter activity, whereas wortmannin (a PI3K inhibitor) did not significantly decrease this activation. By contrast, the LPA-induced inhibitory phosphorylation of GSK $3 \beta$ was marginally decreased by PTX and not by wortmannin, while GF109203 completely inhibited the phosphorylation (Fig. 2). The observation that PTX significantly inhibited the LPA-induced $\beta$-catenin/TCF signaling, with a marginal decrease in the inhibitory phosphorylation of GSK3 $\beta$, 
A

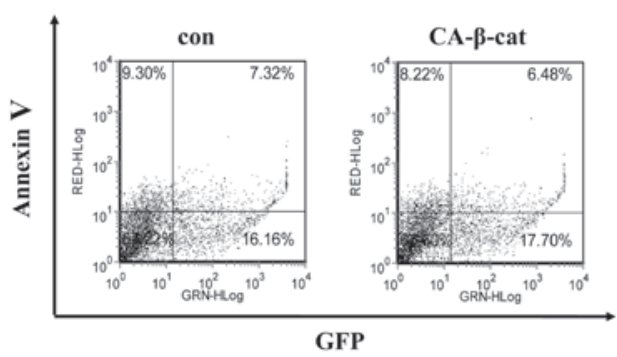

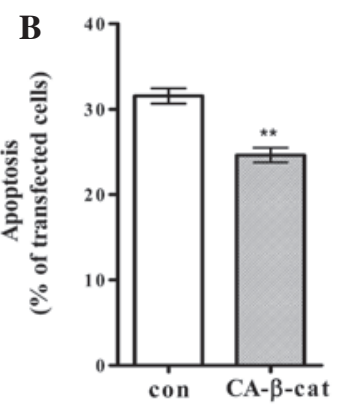

Figure 3. $\beta$-catenin/T cell factor signaling by lysophosphatidic acid (LPA) suppresses apoptosis in differentiating H19-7 cells. (A) Cells were transfected with pCatDt90, a plasmid expressing CA- $\beta$-catenin, or empty vector (control) with phosphorylated enhanced green fluorescent protein (pEGFP). Differentiation was induced and cells were treated with LPA at $39^{\circ} \mathrm{C}$ for $4 \mathrm{~h}$. The cells were harvested, stained with Annexin V conjugated with Alexa Fluor 568 and analyzed by flow cytometry. The intensity of the red fluorescence of Annexin V-Alexa Fluor 568 is shown on the y-axis, the intensity of GFP is shown on the x-axis and the populations of apoptotic cells among cells expressing b-Cat $\Delta 90$ or control cells in the quadrant are shown. Data are representative of two independent experiments. (B) Quantitation of apoptotic cells among the transfected cells with pCatDt90 or empty vector (pcDNA3). Apoptosis was significantly reduced in H19-7 cells transfected with pCatDt90 compared with the control. Results are the mean of triplicate samples from two independent experiments $(\mathrm{n}=6$; ${ }^{* *} \mathrm{P}<0.01$, vs. control).

suggested that LPA may stimulate $\beta$-catenin/TCF transactivation via signaling pathways that are independent of the direct inhibition of GSK3.

Activation of $\beta$-catenin/TCF transcription by LPA suppresses the apoptosis of H19-7 cells. LPA induced cell survival in a dose-responsive manner in the differentiating H19-7 cells; however, the H19-7 cells treated with LPA were not stained with BrdU, suggesting that LPA induced cell survival by protecting H19-7 cells from cell death, not by inducing cell proliferation (data not shown). To determine whether LPA-mediated $\beta$-catenin/TCF signaling was involved in protecting H19-7 cells from cell death, the cells were transfected with a plasmid expressing either CA- $\beta$-catenin or empty vector and a plasmid expressing GFP. Transfected cells differentiated and apoptosis was detected by flow cytometry as described in the Materials and methods. Apoptosis was significantly suppressed in the H19-7 cells expressing CA- $\beta$-catenin compared with that of the control cells (Fig. 3).

\section{Discussion}

Activation of the cognate GPCRs by lipid metabolites, growth factors or a P2Y receptor agonist was shown to activate $\beta$-catenin/TCF signaling, although the mechanism of action is unclear. The GPCR-mediated $\beta$-catenin/TCF transcriptional activation (except for the $\mathrm{P} 2 \mathrm{Y}$ receptor-mediated TCF activation) was demonstrated to be implicated in oncogenicity, including cell proliferation and epithelial-mesenchymal transition in cancer cells, but not in normal physiological processes (18-23). In the present study, it was demonstrated that LPA activated $\beta$-catenin/TCF signaling predominantly via the Gi/o- and PKC-dependent pathways in differentiating HPCs, and that the $\beta$-catenin/TCF signaling induced by LPA was involved in suppressing apoptosis in the HPCs. Activation of $\beta$-catenin/TCF signaling was determined by an increase in the nuclear levels of $\beta$-catenin and the stimulation of TCF reporter activity (Fig. 1). The observation that $\beta$-catenin/TCF transactivation induced the expression of Mcl-1, an anti-apoptotic Bcl2 family member (24), was consistent with a previous study demonstrating that LPA-induced cell survival was mediated by post-translational and transcriptional upregulation of Mcl-1 (2). Whether the direct inhibition of GSK $3 \beta$ is involved in $\beta$-catenin stabilization and subsequent $\beta$-catenin/TCF transactivation remains controversial. LPA was previously shown to induce the inhibitory phosphorylation of GSK $3 \beta$ by PKC, mitogen-activated protein kinase and protein kinase A, but not PI3K, in H19-7 cells (2). Therefore, in the present study, it was investigated whether inhibitory phosphorylation of GSK3 $\beta$ by upstream kinases was involved in the $\beta$-catenin/TCF transactivation. LPA-induced activation of $\beta$-catenin/TCF signaling was significantly inhibited by PTX, although PTX marginally decreased the inhibitory phosphorylation of GSK3 $\beta$. Moreover, the PKC inhibitor decreased the $\beta$-catenin/TCF signaling less potently than that by PTX, although the PKC inhibitor completely abolished the inhibitory phosphorylation of GSK3 $\beta$. Thus, these results suggested that LPA may activate $\beta$-catenin/TCF transactivation via signaling pathways that are independent of the direct inhibition of GSK3. However, $\beta$-catenin/TCF transactivation may have occurred through direct inhibition of GSK3 $\beta$, as the PKC inhibitor significantly decreased the activation of $\beta$-catenin/TCF signaling and the inhibitory phosphorylation of GSK3 $\beta$. Previous studies have suggested that GSK3 $\beta$ only modifies $\beta$-catenin when GSK3 forms the Axin/APC/ $\beta$-catenin destructive complex $(25,26)$, and that only a small pool of GSK3 $\beta$ exists in the Axin scaffolding complex within which GSK3$\beta$ is not readily accessible to upstream kinases (27). Thus, GPCR-mediated activation of $\beta$-catenin/TCF signaling may occur without direct inhibitory phosphorylation of GSK3 $\beta$ in the Axin scaffolding complex, although upstream kinases are involved in this signaling pathway and inhibitory phosphorylation of GSK3 $\beta$ in the other pool is increased. Alternatively to the phosphorylation of GSK3 within the Axin complex, the upstream kinases may activate $\beta$-catenin/TCF signaling by acting on other targets involved in the activation of $\beta$-catenin/TCF signaling, such as 14-3-3-mediated nuclear efflux of $\beta$-catenin by Akt or PKC and KSRP-mediated stabilization of $\beta$-catenin mRNA by PI3K/Akt (28-30). Thus, PKC may activate $\beta$-catenin/TCF signaling without inhibitory phosphorylation of GSK $3 \beta$ in the Axin complex, although the total inhibitory phosphorylation 
of GSK3 3 is increased.

Expression of CA- $\beta$-catenin suppressed apoptosis in differentiating H19-7 cells, suggesting that the activation of the TCF reporter activity by LPA was involved in cell survival of HPCs. Apoptosis of the H19-7 cells was suppressed by $6 \%$ following expression of CA- $\beta$-catenin (Fig. 3). Suppressive effects of $\beta$-catenin/TCF activation on the survival of the H19-7 cells were marginal, but may be physiologically relevant, as small changes in the survival of progenitor pools may produce a marked effect on neurodevelopment. It was suggested that only a $3.5 \%$ increase in survival and a $2.5 \%$ increase in terminal mitosis of progenitor cells induced an $\sim 30 \%$ increase in the thickness of the cerebral cortical wall (13). In concordance with the present results, previous studies demonstrated that $\beta$-catenin/TCF signaling was involved in the survival of neural precursors, although the molecular mechanisms remain unknown (31-33). In conclusion, the results suggested that LPA may regulate $\beta$-catenin/TCF signaling in NPCs, as LPA is involved in neurogenesis by controlling the survival of neural precursors.

\section{Acknowledgements}

This study was supported by the National Research Foundation of Korea Grant funded by the Korean Government (grant no. 2010-0005848), and by the Priority Research Centers Program through the National Research Foundation of Korea (NRF), funded by the Ministry of Education, Science and Technology (grant no. NRF-2009-0094071).

\section{References}

1. Anliker B and Chun J: Lysophospholipid G protein-coupled receptors. J Biol Chem 279: 20555-20558, 2004.

2. Sun Y, Nam JS, Han DH, et al: Lysophosphatidic acid induces upregulation of Mcl-1 and protects apoptosis in a PTX-dependent manner in H19-7 cells. Cell Signal 22: 484-494, 2010.

3. Zhang L, Yang X, Yang S and Zhang J: The Wnt/ $\beta$-catenin signaling pathway in the adult neurogenesis. Eur J Neurosci 33: $1-8,2011$.

4. Ille F, Atanasoski S, Falk S, et al: Wnt/BMP signal integration regulates the balance between proliferation and differentiation of neuroepithelial cells in the dorsal spinal cord. Dev Biol 304: 394-408, 2007.

5. Zhang J, Woodhead GJ, Swaminathan SK, et al: Cortical neural precursors inhibit their own differentiation via $\mathrm{N}$-cadherin maintenance of beta-catenin signaling. Dev Cell 18: 472-479, 2010.

6. Wang X, Kopinke D, Lin J, et al: Wnt signaling regulates postembryonic hypothalamic progenitor differentiation. Dev Cell 23: 624-636, 2012

7. Tang M, Villaescusa JC, Luo SX, et al: Interactions of Wnt/beta-catenin signaling and sonic hedgehog regulate the neurogenesis of ventral midbrain dopamine neurons J Neurosci 30: 9280-9291, 2010.

8. Pöschl J, Grammel D, Dorostkar MM, Kretzschmar HA and Schüller U: Constitutive activation of $\beta$-catenin in neural progenitors results in disrupted proliferation and migration of neurons within the central nervous system. Dev Biol 374 319-332, 2013

9. Zhao S, Fu J, Liu X, Wang T, Zhang J and Zhao Y: Activation of Akt/GSK-3beta/beta-catenin signaling pathway is involved in survival of neurons after traumatic brain injury in rats. Neurol Res 34: 400-407, 2012.

10. Pérez-Álvarez MJ, Maza Mdel C, Anton M, Ordoñez L and Wandosell F: Post-ischemic estradiol treatment reduced glial response and triggers distinct cortical and hippocampal signaling in a rat model of cerebral ischemia. J Neuroinflammation 9: 157, 2012
11. Mavila N, James D, Utley S, et al: Fibroblast growth factor receptor-mediated activation of AKT- $\beta$-catenin-CBP pathway regulates survival and proliferation of murine hepatoblasts and hepatic tumor initiating stem cells. PloS One 7: e50401, 2012.

12. Lei ZN, Liu F, Zhang LM, Huang YL and Sun FY: Bcl-2 increases stroke-induced striatal neurogenesis in adult brains by inhibiting BMP-4 function via activation of $\beta$-catenin signaling. Neurochem Int 61: 34-42, 2012.

13. Kingsbury Ma, Rehen SK, Contos JJ, Higgins CM and Chun J: Non-proliferative effects of lysophosphatidic acid enhance cortical growth and folding. Nat Neurosci 6: 1292-1299, 2003.

14. Valenta T, Hausmann $G$ and Basler K: The many faces and functions of $\beta$-catenin. EMBO J 31: 2714-2736, 2012.

15. Jin T, George Fantus I and Sun J: Wnt and beyond Wnt: multiple mechanisms control the transcriptional property of beta-catenin. Cell Signal 20: 1697-1704, 2008.

16. Eves EM, Boise LH, Thompson CB, Wagner AJ, Hay N and Rosner MR: Apoptosis induced by differentiation or serum deprivation in an immortalized central nervous system neuronal cell line. J Neurochem 67: 1908-1920, 1996.

17. Rhee HJ, Nam JS, Sun Y, et al: Lysophosphatidic acid stimulates cAMP accumulation and cAMP response element-binding protein phosphorylation in immortalized hippocampal progenitor cells. Neuroreport 17: 523-526, 2006.

18. Yang M, Zhong WW, Srivastava N, et al: G protein-coupled lysophosphatidic acid receptors stimulate proliferation of colon cancer cells through the $\beta$-catenin pathway. Proc Natl Acad Sci USA 102: 6027-6032, 2005 .

19. Desbois-Mouthon C, Cadoret A, Blivet-Van Eggelpoël MJ, et al: Insulin and IGF-1 stimulate the beta-catenin pathway through two signalling cascades involving GSK-3beta inhibition and Ras activation. Oncogene 20: 252-259, 2001.

20. Castellone MD, Teramoto H, Williams BO, Druey KM and Gutkind JS: Prostaglandin E2 promotes colon cancer cell growth through a Gs-axin-beta-catenin signaling axis. Science 310: 1504-1510, 2005

21. Verras M and Sun Z: Beta-catenin is involved in insulin-like growth factor 1-mediated transactivation of the androgen receptor. Mol Endocrinol 19: 391-398, 2005.

22. Ortega F, Pérez-Sen R and Miras-Portugal MT: Gi-coupled P2Y-ADP receptor mediates GSK-3 phosphorylation and beta-catenin nuclear translocation in granule neurons. J Neurochem 104: 62-73, 2008

23. Yi F, Sun J, Lim GE, Fantus IG, Brubaker PL and Jin T: Cross talk between the insulin and Wnt signaling pathways: evidence from intestinal endocrine L cells. Endocrinology 149: 2341-2351, 2008.

24. Iqbal S, Zhang S, Driss A, et al: PDGF upregulates Mcl-1 through activation of $\beta$-catenin and HIF- $1 \alpha$-dependent signaling in human prostate cancer cells. PloS One 7: e30764, 2012.

25. Hur EM and Zhou FQ: GSK3 signalling in neural development. Nat Rev Neurosci 11: 539-551, 2010.

26. Wu D and Pan W: GSK3: a multifaceted kinase in Wnt signaling. Trends Biochem Sci 35: 161-168, 2010.

27. Ng SS, Mahmoudi T, Danenberg E, et al: Phosphatidylinositol 3-kinase signaling does not activate the wnt cascade. J Biol Chem 284: 35308-35313, 2009.

28. Gherzi R, Trabucchi M, Ponassi M, et al: The RNA-binding protein KSRP promotes decay of beta-catenin $\mathrm{mRNA}$ and is inactivated by PI3K-AKT signaling. PLoS Biol 5: e5, 2006.

29. Takemaru K, Fischer V and Li FQ: Fine-tuning of nuclear-catenin by Chibby and 14-3-3. Cell Cycle 8: 210-213, 2009.

30. Luna-Ulloa LB, Hernández-Maqueda JG, Santoyo-Ramos P, Castañeda-Patlán MC and Robles-Flores M: Protein kinase C $\xi$ is a positive modulator of canonical Wnt signaling pathway in tumoral colon cell lines. Carcinogenesis 32: 1615-1624, 2011.

31. Paek H,Hwang JY,Zukin RS and Hébert JM: $\beta$-Catenin-dependent FGF signaling sustains cell survival in the anterior embryonic head by countering Smad4. Dev Cell 20: 689-699, 2011.

32. Holowacz T, Huelsken J, Dufort D and van der Kooy D: Neural stem cells are increased after loss of $\beta$-catenin, but neural progenitors undergo cell death. Eur J Neurosci 33: 1366-1375, 2011.

33. Chen BY, Wang X, Wang ZY, Wang YZ, Chen LW and Luo ZJ: Brain-derived neurotrophic factor stimulates proliferation and differentiation of neural stem cells, possibly by triggering the Wnt/ $\beta$-catenin signaling pathway. J Neurosci Res 91: 30-41, 2013. 